\title{
Hemodynamic changes acutely determined by primary PCI in STEMI patients evaluated with a minimally invasive method
}

\author{
Cristina Giglioli $^{1 *}$, Omar Tujjar ${ }^{2}$, Emanuele Cecchi ${ }^{1}$, Daniele Landi ${ }^{1}$, Marco Chiostri ${ }^{2}$, \\ Serafina Valente ${ }^{1}$, Giorgio Jacopo Baldereschi ${ }^{1}$, Francesco Meucci ${ }^{1}$, Gian Franco Gensini ${ }^{2}$, \\ Salvatore Mario Romano ${ }^{2}$
}

${ }^{1}$ Department of Heart and Vessels, Azienda Ospedaliero-Universitaria Careggi, Florence, Italy

${ }^{2}$ Department of Critical Care and Surgery, Unit of Internal Medicine and Cardiology, University of Florence, Italy

Email: ${ }^{*}$ cristinagiglioli@yahoo.it

Received 10 April 2013; revised 10 May 2013; accepted 24 May 2013

Copyright (C) 2013 Cristina Giglioli et al. This is an open access article distributed under the Creative Commons Attribution License, which permits unrestricted use, distribution, and reproduction in any medium, provided the original work is properly cited.

\begin{abstract}
Objective: Few studies are available on the hemodynamic changes acutely determined by Primary Percutaneous Coronary Intervention (PCI) in ST-Elevation Myocardial Infarction (STEMI) patients, probably for the difficult evaluation of hemodynamic variables in this acute setting. Therefore, the paper is to evaluate the variations of several hemodynamic parameters determined by PCI using PRAM (Pressure Recording Analytical Method), minimally invasive hemodynamic monitoring. Methods: We analyzed in 20 STEMI patients submitted to PCI several hemodynamic variables, assessed with PRAM from radial/ femoral artery, 3-minute before PCI and at endprocedure. Variables measures were: systolic, diastolic, dicrotic and mean arterial pressures; heart rate (HR); stroke volume (SV); systemic vascular resistance (SVR); dP/dt $t_{\text {max }}$; cardiac cycle efficiency (CCE). Results: In our patients $\mathrm{HR}$, SVR and $\mathbf{d P / d t} t_{\max }$ decreased significantly $(85 \pm 6.3$ to $77 \pm 4.5, p=0.002$; $1738 \pm 241$ to $1450 \pm 253, p=0.022 ; 1.22 \pm 0.11$ to 1.11 $\pm 0.12, p=0.007$, respectively) while $\mathrm{CCE}$ and $\mathrm{SV}$ increased significantly $(-0.25 \pm 0.23$ to $-0.01 \pm 0.12$, p $<0.001 ; 53 \pm 8.4$ to $65 \pm 11.2, p<0.001$, respectively). Conclusions: Hemodynamic monitoring with PRAM seems feasible during primary PCI and can provide further notions regarding the acute effects determined on cardiovascular system by the culprit artery revascularization. The most significant hemodynamic changes acutely observed in our study should be mainly ascribed to the reduction in sympathetic activity after PCI with a rapid improvement of the car-
\end{abstract}

\footnotetext{
Corresponding author.
}

diovascular system efficiency.

Keywords: STEMI; Primary PCI; Hemodynamic Monitoring; Cardiac Output

\section{INTRODUCTION}

Primary Percutaneous Coronary Intervention (PCI) is considered the gold standard for the treatment of STElevation Myocardial Infarction (STEMI) because it is able to reduce short and long-term mortality and Major Adverse Cardiac Events at follow-up.

Although in the last fifteen years Primary PCI has become a widespread treatment for STEMI patients and several studies have been performed on different aspects related to this procedure, few data are available about the hemodynamic changes that Primary PCI can acutely determine with the culprit artery re-opening. Lack of data can be mainly ascribed to the difficulty of performing, in the setting of STEMI and during an urgent and invasive procedure such as Primary PCI, a "classic" hemodynamic monitoring with a Swan-Ganz catheter. In fact, pulmonary artery catheterization is invasive and can be associated with severe complications, it is a time-consuming procedure [1] and during instable patient conditions it could be not able to estimate the cardiac output (CO) [2-4].

The minimally invasive hemodynamic monitoring systems, mainly based on the Pulse Contour Method, could be more suitable for the evaluation of the acute effects of Primary PCI. In this brief communication we report our preliminary experience, with one of these monitoring systems, PRAM (Pressure Recording Analytical Method) $[5,6]$ used in 20 STEMI patients during primary PCI in 
order to evaluate the hemodynamic changes is determined by the culprit vessel opening.

\section{METHODS}

Patients analyzed were consecutively admitted from January to March 2011 to the Catheterization Laboratory of the University of Florence, within 12 hours from symptoms onset. Informed consent was obtained from each patient and the study protocol conforms to the ethical guidelines of the 1975 Declaration of Helsinki as reflected in a priori approval by the institution's human research committee.

Clinical baseline characteristics of patients investigated are reported in Table 1.

Primary PCI was performed by femoral $(\mathrm{n}=9)$ or radial $(\mathrm{n}=11)$ approach with standard technique. Before PCI all patients received Morphine Chlorohydrate 5 - 10 mg i.v. for chest pain control, Unfractioned Heparin (70 $\mathrm{IU} / \mathrm{kg}$ ), Acetylsalicylic acid $325 \mathrm{mg}$ and Clopidogrel 600 $\mathrm{mg}$.

Glycoprotein IIb/IIIa inhibitors, thrombus aspiration devices and the type of stent implanted (bare metal or drug eluting stent) was at attending physician judgment.

Arterial waveform was sampled at $1000 \mathrm{~Hz}$ by PRAM monitoring (MostCare ${ }^{\circledR}$, Vytech, Padova, Italy), (and data electronically stored) directly through the sheath used for PCI. Following radial or femoral artery cannulation, in addition to the standard monitoring for PCI, all patients underwent hemodynamic monitoring by means of PRAM. This system applies, beat by beat, the mathematical analysis of pressure waveform, after careful check of the signal pressure quality, to obtain hemodynamic parameters [7]. At variance with other Pulse Con-

Table 1. Baseline characteristics of patients investigated.

\begin{tabular}{cc}
\hline Males/Females & $13 / 7$ \\
Age (years) & $66 \pm 13$ \\
Infarct location: anterior/inferior & $11 / 9$ \\
Symptoms onset-cath lab (min) & $344 \pm 388$ \\
CK-MB (ng/ml) & $141 \pm 129$ \\
TnI (ng/ml) & $56 \pm 50$ \\
Angiographic EF (\%) & $38.4 \pm 8.5$ \\
Death & 1 \\
Killip Class & 11 \\
I & 6 \\
II & 1 \\
III & 2 \\
IV & \\
\hline
\end{tabular}

CK-MB: creatine kinase MB; TnI: troponin I; EF: ejection fraction. tour Methods (PCM), based on a calibration and/or preestimated data obtained from other subjects and applied to the patient examined [8-10], PRAM uses directly the pressure wave of the patient under evaluation allowing an analysis more in keeping with the patient under study [5].

It is noteworthy that not only the system analyses the pressure curve during systolic phase, but considers also the diastolic phase and takes into account the whole pressure value during heartbeat: the pulsatory and the continuous contributions. Although several analogies can be noted between this method and other PCMs, however, PRAM, for the first time, introduced variables obtained during the whole cardiac cycle, without pre-calibrations and/or pre-estimated data. Data are then applied to the investigated patient by the $\mathrm{P} / \mathrm{t}$ of each point of the whole cardiac cycle. The instantaneous power per unit area of the considered vessel can be assessed, multiplying it by a function related to the expected and the measured mean pressure [11].

Hemodynamic variables obtained with this monitoring system are: systolic, diastolic, dicrotic and mean arterial blood pressure; heart rate (HR); cardiac output (CO); stroke volume (SV) and systemic vascular resistance (SVR); $\mathrm{dP} / \mathrm{dt}_{\max }$; cardiac cycle efficiency (CCE).

CCE is a novel hemodynamic parameter which indicates hemodynamic performance in terms of energy expenditure that mirrors the systemic cardiovascular status, including the dynamic interplay of all systems/factors that are involved in the homeostasis of each single beat [11]. PRAM allows to measure CCE because it is able to estimate $\mathrm{CO}$ [as the product of SV and HR] by means of the dynamic impedance of the patient in vivo, which is subjected to continuous changes (from beat to beat and within a single beat).

This efficiency is obtained through the ratio of the two hemodynamic works: systolic energetic performance/total energetic expenditure of the heartbeat. Thus, CCE represents the energetic expenditure performed by the cardiovascular system to maintain hemodynamic balance. It is possible to assess the correlation between different energetic expenditures and CCE as the expression of the ability of the cardiovascular system to maintain homeostasis at different energetic levels resulting from simultaneous interactions between the pump function (both mechanical and electrical contributions), the arterial system, venous return and the pulmonary circulation.

Hemodynamic monitoring by PRAM system was started before PCI and stopped with the arterial sheath removal at the end of the procedure (mean duration 85 min). Data reported in this communication refer to the evaluation performed 3 min before Primary PCI and 3 min before the end of the procedure with a careful assessment of the arterial pressure waveform in order to 
avoid over-underdamping artifacts $[7,12]$.

Data were stored in a dedicated database and analyzed with SPSS 13.0 for Windows statistical software (SPSS Inc, Chicago, IL). Statistical significance was fixed at $\mathrm{P}$ $<0.05$. Correlation between CCE values and Ejection Fraction (EF) values was assessed with linear regression analysis.

\section{RESULTS}

Primary PCI was effective in all patients and a TIMI III flow was obtained in the overall population.

Thrombus aspiration devices were used in 18 patients (90\%). During PCI, two patients had ventricular fibrillation interrupted with DC shock, 3 patients with inferior STEMI had severe hypotension treated with fluid and atropine administration, 1 patient experienced pulmonary edema and 1 required intra-aortic balloon pump positioning for severe hemodynamic instability. The main hemodynamic variables recorded with PRAM before and after Primary PCI are reported in Table 2. In our group of patients after PCI with respect to before HR, SVR and $\mathrm{dP} / \mathrm{dt}_{\max }$ decreased significantly while CCE and SV increased significantly (Table 2). A positive significant correlation was found between angiographic EF and CCE evaluated at the end of Primary PCI (Figure 1).

\section{DISCUSSION}

In the present study, we analyzed the hemodynamic variations determined by Primary angioplasty in the setting of STEMI by means of PRAM, a minimally invasive method. Our analyses showed after culprit artery reopening a significant decrease in $\mathrm{HR}, \mathrm{SVR}$ and $\mathrm{dP} / \mathrm{dt}_{\max }$

Table 2. Hemodynamic parameters obtained by PRAM (mean $\pm \mathrm{SD}$ ) before and after Primary PCI.

\begin{tabular}{cccc}
\hline & Before PCI & Post PCI & p value \\
\hline Diastolic pressure (mmHg) & $74 \pm 5.2$ & $72 \pm 6.6$ & 0.362 \\
Systolic pressure (mmHg) & $139 \pm 8.1$ & $135 \pm 11.8$ & 0.186 \\
$\begin{array}{c}\text { Dictrotic pressure (mmHg) } \\
\text { Mean arterial pressure }\end{array}$ & $96 \pm 7.3$ & $92 \pm 8.9$ & 0.153 \\
(mmHg) & $97 \pm 5.5$ & $93 \pm 8.2$ & 0.137 \\
HR (bpm) & $85 \pm 6.3$ & $77 \pm 4.5$ & $0.002^{*}$ \\
SVR (dyne*sec/cm $)$ & $1738 \pm 241.2$ & $1450 \pm 253.4$ & $0.022^{*}$ \\
CCE (units) & $-0.25 \pm 0.23$ & $-0.01 \pm 0.12$ & $<0.001^{*}$ \\
CO (1/min) & $4.50 \pm 0.78$ & $4.92 \pm 0.69$ & 0.076 \\
SV (ml) & $53.1 \pm 8.4$ & $65 \pm 11.2$ & $<0.001^{*}$ \\
$\mathrm{dP}^{2} \mathrm{dt}_{\max }(\mathrm{mmHg} / \mathrm{msec})$ & $1.22 \pm 0.11$ & $1.11 \pm 0.12$ & $0.007^{*}$ \\
\hline
\end{tabular}

HR: heart rate; SVR: systemic vascular resistance; CCE: cardiac cycle efficiency; CO: cardiac output; SV: stroke volume. ${ }^{*} \mathrm{p}<0.05$.

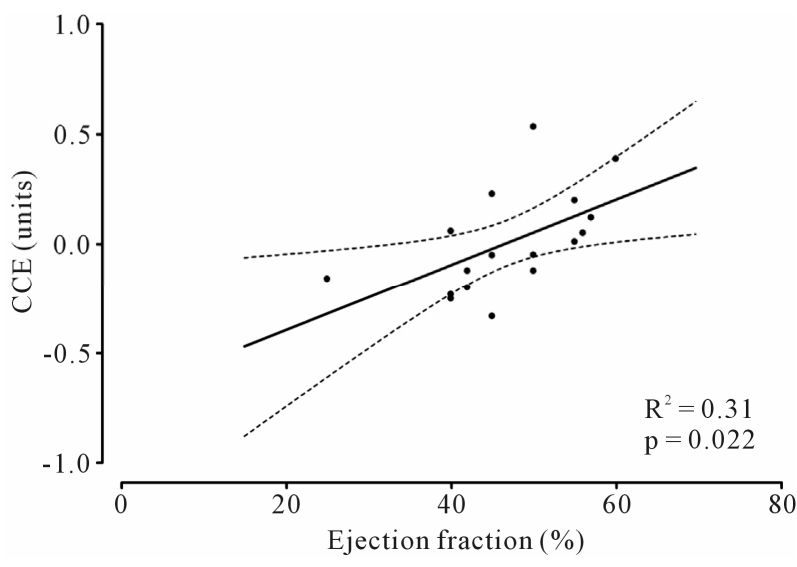

Figure 1. Correlation between cardiac cycle efficiency (CCE) and angiographic ejection fraction (EF) assessed at the end of percutaneous coronary intervention (PCI).

as well as a significant increase in CCE and SV without any significant variation in $\mathrm{CO}$.

These preliminary results deserve some considerations.

The re-opening of the culprit vessel is immediately associated with a significant reduction of HR and SVR that can be ascribed, at least in part, to the cardiac performance improvement due to reperfusion even though it is supposable that these hemodynamic effects could be mainly determined by the reduction in sympathetic nervous system activity that usually increases in the acute phase of myocardial infarction $[13,14]$, as a compensatory mechanism.

Similar observations can be drawn from the trend of $\mathrm{dP} / \mathrm{dt}_{\max }$, a parameter that mirrors myocardial contractility whose normal values range between 0.9 - $1 \mathrm{mmHg} / \mathrm{msec}$ $[12,15]$. In our patients values of $\mathrm{dP} / \mathrm{dt}_{\max }$ were high either before and after PCI (Table 2) with a significant reduction after the procedure. We interpreted this reduction in $\mathrm{dP} / \mathrm{dt}_{\max }$ at the end of PCI not as a negative effect of the procedure but as a return to normal values due to the decrease in sympathetic activity, usually high in the acute phase of myocardial infarction. Therefore, we can suppose that, despite a possible improvement in cardiac performance, we did not observe any increase in $\mathrm{dP} / \mathrm{dt}_{\max }$ because this parameter was excessively high before PCI.

The re-opening of the culprit vessel is immediately associated also with a significant increase in SV and in CCE but not in CO. The increase in SV can be ascribed to the reduction of $\mathrm{HR}$ and SVR as well as to the improvement of cardiovascular performance consequent to reperfusion. However, $\mathrm{CO}$ did not significantly increase because it is the product of SV that increased and HR that decreased in our patients. Although $\mathrm{CO}$ is a hemodynamic parameter of great importance, its significance can be limited by its dependence on other variables such as HR and SV. Notably, in our group of patients CCE 
increased significantly after PCI and this parameter showed also a direct significant correlation with the angiographic EF evaluated at the end of the procedure.

The CCE could determine whether a "predetermined" individual hemodynamic status is achieved by compensatory mechanisms. One could then get information on the "reserve" of the patient under evaluation. Thus, variations in every patient can be followed to determine whether a disease or a therapeutic intervention has positively or negatively influenced the "efficiency" of the cardiovascular system.

Therefore, in this preliminary experience, CCE would seem to be, in the early phase, an index more sensitive than $\mathrm{CO}$ in demonstrating an improvement of cardiovascular performance after myocardial reperfusion.

\section{CONCLUSION}

In conclusion, the hemodynamic monitoring by means of PRAM seems feasible during primary PCI in STEMI patients, and can provide further notions regarding the acute effects determined on the cardiovascular system by the culprit artery revascularization. The most significant hemodynamic changes acutely observed in our preliminary study should be mainly ascribed to the reduction in sympathetic activity after culprit artery re-opening with a consequent rapid improvement of the energetic expenditure in the cardiovascular system.

\section{REFERENCES}

[1] Chatterjee, K. (2009) The Swan-Ganz catheters: Past, present, and future. A viewpoint. Circulation, 119, 147152. doi:10.1161/CIRCULATIONAHA.108.811141

[2] Mackenzie, J.D., Haites, N.E. and Rawles, J.M. (1986) Method of assessing the reproducibility of blood flow measurement: Factor influencing the performance of thermodilution cardiac output computers. British Heart Journal, 55, 14-24. doi:10.1136/hrt.55.1.14

[3] Jonson, R.W. and Normann, R.A. (1989) Central venous blood temperature fluctuations and thermodilution signal processing in dogs. Annals of Biomedical Engineering, 17, 657-669. doi:10.1007/BF02367469

[4] Reuter, D.A., Huang, C., Edrich, T., Shernan, S.K. and Eltzsching, H.K. (2010) Cardiac output monitoring using indicator-dilution techniques: Basics, limits, and prospectives. Anesthesia \& Analgesia, 110, 799-811. doi:10.1213/ANE.0b013e3181cc885a

[5] Romano, S.M. and Pistolesi, M. (2002) Assessment of cardiac output from systemic arterial pressure in humans. Critical Care Medicine, 30, 1834-1841. doi:10.1097/00003246-200208000-00027

[6] Zangrillo, A., Maj, G., Monaco, F., Scandroglio, A.M.,
Nuzzi, M., Plumari, V., Virzo, I., Bignami, E., Casiraghi, G. and Landoni, G. (2010) Cardiac index validation using the pressure recording analytic method in unstable patients. Journal of Cardiothoracic and Vascular Anesthesia, 24, 265-269. doi:10.1053/j.jvca.2009.09.019

[7] Romagnoli, S., Romano, S.M., Bevilacqua, S., Lazzeri, C., Gensini, G.F., Pratesi, C., Quattrone, D., Dini, D. and De Gaudio, A.R. (2011) Dynamic response of liquidfilled catheter systems for measurement of blood pressure: Precision of measurements and reliability of pressure recording analytical method with different disposable system. Journal of Critical Care, 26, 415-422. doi:10.1016/j.jcrc.2010.08.010

[8] Gödje, O., Hoke, K., Goetz, A.E., Felbinger, T.W., Reuter, D.A., Reichart, B., Friedl, R., Hannekum, A. and Pfeiffer, U.J. (2002) Reliability of a new algorithm for continuous cardiac output determination by pulse-contour analysis during hemodynamic instability. Critical Care Medicine, 30, 52-58.

doi:10.1097/00003246-200201000-00008

[9] Pittman, J., Bar, Y.S., Sum, P.J., Sherwood, M. and Mark, M. (2005) Continuous cardiac output monitoring with pulse contour analysis: A comparison with lithium indicator dilution cardiac output measurement. Critical Care Medicine, 30, 2015-2021. doi:10.1097/01.CCM.0000179021.36805.1F

[10] Romagnoli, S. and Romano, S.M. (2010) Estimation of hemodynamic parameters by arterial waveform: Available technologies. Anesthesia \& Analgesia, 110, 257-258. doi:10.1213/ANE.0b013e3181c135f1

[11] Romano, S.M. (2012) Cardiac cycle efficiency: A new parameter able to fully evaluate the dynamic interplay of the cardiac circulatory system. International Journal of Cardiology, 155, 326-327. doi:10.1016/j.ijcard.2011.12.008

[12] Giglioli, C., Landi, D., Cecchi, E., Chiostri, M., Gensini, G.F., Valente, V., Ciaccheri, M., Castelli, G. and Romano, S.M. (2011) Effects of ultrafiltration vs. diuretics on clinical, biohumoral and hemodynamic variables in patients with decompensated heart failure: The ULTRA-DISCO study. European Journal of Heart Failure, 13, 337-346. doi:10.1093/eurjhf/hfq207

[13] Ceremuzynski, L. (1981) Hormonal and metabolic reactions evoked by acute myocardial infarction. Circulation Research, 48, 767. doi:10.1161/01.RES.48.6.767

[14] Karlsberg, R.P., Cryer, P.E. and Roberts, R. (1981) Serial plasma cathecolamine response early in the course of clinical acute myocardial infarction: relationship to infarct extent and mortality. American Heart Journal, 102, 24-29. doi:10.1016/0002-8703(81)90408-7

[15] Givertz, M.M., Andreou, C., Conrad, C.H. and Colucci, W.S. (2007) Direct myocardial effects of levosimendan in humans with left ventricular dysfunction: Alteration of force-frequency and relaxation-frequency relationships. Circulation, 115, 1218-1224. 